\title{
Construction of Resolvable Spatial Row-Column Designs
}

\author{
E. R. Williams, ${ }^{1, *}$ J. A. John, ${ }^{2}$ and D. Whitaker ${ }^{2}$ \\ ${ }^{1}$ CSIRO Forestry and Forest Products, P.O. Box E4008, Kingston, ACT 2604, Australia \\ ${ }^{2}$ Department of Statistics, University of Waikato, Private Bag 3105, Hamilton, New Zealand \\ * email: Emlyn.Williams@csiro.au
}

\begin{abstract}
Summary. Resolvable row-column designs are widely used in field trials to control variation and improve the precision of treatment comparisons. Further gains can often be made by using a spatial model or a combination of spatial and incomplete blocking components. Martin, Eccleston, and Gleeson (1993, Journal of Statistical Planning and Inference 34, 433-450) presented some general principles for the construction of robust spatial block designs which were addressed by spatial designs based on the linear variance (LV) model. In this article we define the two-dimensional form of the LV model and investigate extensions of the Martin et al. principles for the construction of resolvable spatial row-column designs. The computer construction of efficient spatial designs is discussed and some comparisons made with designs constructed assuming an autoregressive variance structure.
\end{abstract}

KEY WORDS: Average efficiency factor; Deviance; Latinized designs; Linear variance; Residual maximum likelihood; Resolvable designs; Row-column designs; Spatial designs.

\section{Introduction}

In field trials it is essential to effectively accommodate field trend so that it does not influence treatment comparisons. Efficient experimental designs are used to maximize the separation of treatment information from field variation. Lattice designs (Cochran and Cox, 1957) have traditionally provided a convenient class of resolvable incomplete block designs for use in field trials. In more recent times, the computer generation of generalized lattice designs has provided a wide range of efficient block designs (John and Williams, 1995). If the plots of a trial are laid out in a two-dimensional arrangement, then resolvable row-column designs can be used. These designs allow for adjustment for field trend in two directions. The software package CycDesigN (Whitaker, Williams, and John, 2002) provides a convenient facility for constructing efficient resolvable row-column designs.

An alternative to classical blocking for the control of field variation is to use spatial design and analysis. Spatial or neighbor methods for the analysis of field trials have been available since Papadakis (1937). There have been a number of different approaches to modeling spatial trend for various field layouts, notably the discussion article by Wilkinson et al. (1983). Williams (1986) introduced the linear variance (LV) model for one-dimensional spatial analysis which corresponds to the first-difference model of Besag and Kempton (1986). Gleeson and Cullis (1987) presented a one-dimensional autoregressive (AR) model for spatial analysis and extended this to two dimensions in Cullis and Gleeson (1991).

The relative merits of spatial models versus more conventional incomplete blocking (IB) have been discussed at length; see, for example, Wilkinson et al. (1983). Williams (1986) reanalyzed the set of 166 variety trials considered by
Patterson and Hunter (1983) and reported gains in efficiency of $36 \%$ and $79 \%$ for the LV model over the IB and randomized complete block (RCB) models, respectively. Baird and Mead (1991) conducted some detailed simulation studies of spatial and incomplete block models. Kempton, Seraphin, and Sword (1994) carried out a comparison of two-dimensional spatial analysis with row-column analysis on 224 U.K. cereal trials and reported gains in efficiency of $59 \%$ and $53 \%$ over RCB analysis, respectively.

The construction of efficient spatial block designs has been investigated by several authors; see, for example, Wild and Williams (1987) and Martin and Eccleston (1991). Martin, Eccleston, and Gleeson (1993) showed that spatial block designs derived from the one-dimensional LV model are robust over a range of parameter values and alternate decay models such as AR; they developed some principles for the construction of robust spatial block designs and noted that the principles accord with designs that are efficient for the LV model. One advantage of using the LV model for the purpose of design construction is that the variance matrix can be written as a linear combination of known matrices with parameter coefficients. That is, there are no unknown correlation parameters within these matrices.

The purpose of this article is to provide a framework for the construction of robust resolvable spatial row-column designs and investigate the ideas of Martin et al. (1993) in two dimensions. To do this we first define the two-dimensional form of the LV model, note some of its properties, and give an example. Then in Section 3, we develop a criterion for the construction of resolvable spatial row-column designs using the two-dimensional LV model. We also discuss the computer construction of two-dimensional spatial designs by generalizing 
methods that have been developed for directly updating the average efficiency factor of incomplete block designs (John and Whitaker, 2000).

\section{Two-Dimensional LV Model}

We assume a resolvable design for $v$ treatments and with $r$ replicates, each comprising $k$ rows and $s$ columns, where $v=$ $k s$. If $y$ is an $n \times 1$ vector of mean-corrected yields $(n=v r)$, then we define the model

$$
\begin{aligned}
\mathrm{E}(y) & =R \pi+X \tau \\
\operatorname{var}(y) & =\sigma^{2}\left(I_{n}-P_{G}\right) V\left(I_{n}-P_{G}\right),
\end{aligned}
$$

where $\pi$ and $\tau$ are $r \times 1$ and $v \times 1$ vectors of replicate and treatment effects, respectively, $R$ and $X$ are the corresponding design matrices, $P_{G}$ is the projection matrix for the grand mean, that is, $P_{G}=K_{n}$ where $K_{n}$ is the $n \times n$ matrix with every element equal to $1 / n$, and $V$ is given by

$V=I_{n}+\eta_{R} P_{R}+\eta_{C} P_{C}-\left(\phi_{R} F_{R}+\phi_{C} F_{C}+\phi_{R C} F_{R C}\right)$,

where the $\eta_{R}, \eta_{C}, \phi_{R}, \phi_{C}$, and $\phi_{R C}$ are parameters to be estimated; $P_{R}$ and $P_{C}$ are projection matrices for rows and columns, respectively, that is, assuming the data are ordered column by column then $P_{R}=I_{r} \otimes K_{s} \otimes I_{k}$ and $P_{C}=I_{r} \otimes$ $I_{s} \otimes K_{k} ; F_{R}, F_{C}$, and $F_{R C}$ are $\mathrm{LV}$ matrices for rows, columns, and plots not in the same row or column, respectively. In particular, $F_{R}=I_{r} \otimes U_{s} \otimes I_{k}, F_{C}=I_{r} \otimes I_{s} \otimes U_{k}$, and $F_{R C}=$ $I_{r} \otimes U_{s} \otimes U_{k}$, where $U_{k}=3 L_{k} /\left(k^{2}-1\right)$ and $L_{k}$ is a $k \times$ $k$ matrix with $(i, j)$ th element given by $|i-j|$. Note that $P_{R} F_{R} P_{R}=P_{R}$ with a similar relation for columns. We call this the two-dimensional $\mathrm{LV}(\mathrm{LV}+\mathrm{RC})$ model and make the following observations:

(i) The specification uses the concept of a separable process (Martin, 1979). Cullis and Gleeson (1991) have also used this structure in their formation of a two-dimensional AR model.

(ii) The pairwise variance between plots $i_{1}$ and $i_{2}$ in the same column of a replicate is

$$
2 \sigma^{2}\left\{1+\eta_{R} / s+3 \phi_{R}\left|i_{1}-i_{2}\right| /\left(k^{2}-1\right)\right\}
$$

and for plots $j_{1}$ and $j_{2}$ in the same row of a replicate this becomes

$$
2 \sigma^{2}\left\{1+\eta_{C} / k+3 \phi_{C}\left|j_{1}-j_{2}\right| /\left(s^{2}-1\right)\right\} .
$$

The pairwise variance between two plots not in the same row or column but in the same replicate is

$$
\begin{aligned}
2 \sigma^{2} & {\left[1+\eta_{R} / s+\eta_{C} / k+9 \phi_{R C}\left|i_{1}-i_{2}\right|\left|j_{1}-j_{2}\right| /\right.} \\
& \left.\times\left\{\left(k^{2}-1\right)\left(s^{2}-1\right)\right\}\right] .
\end{aligned}
$$

(iii) The $\mathrm{LV}+\mathrm{RC}$ model retains some of the features of the one-dimensional LV + IB model of Williams (1986) in that if a spatial effect is not evident then we can set $\phi_{R}, \phi_{C}$, and $\phi_{R C}$ to zero and the model reduces to the standard mixed-model form for a resolvable row-column design. Alternatively, in the situation where spatial components are required, the model may be just as effective without row and/or column effects.

(iv) The $\mathrm{LV}+\mathrm{RC}$ model includes an independent error term or nugget effect. It is usually necessary to include this term; for example, Williams (1986) noted that in the LV + IB analyses of the Patterson and Hunter (1983) data only 5 out of 166 data sets did not require an independent error term. For forestry trials where treatments are mainly from outbred populations the independent error term is likely to be much larger than is seen in the analysis of other field crops.

(v) Williams (1986) equated mean squares to expectation in order to estimate parameters for the LV + IB model. Nowadays, parameter estimation can be very conveniently carried out using residual maximum likelihood (REML) in major statistical packages and this method is recommended for the $\mathrm{LV}+\mathrm{RC}$ model. The deviance of the fitted model can be used to determine whether random terms can be dropped from the model (Welham and Thompson, 1997). In many cases we have found that the last term in (2) can be excluded, because $\phi_{R C}$ is estimated as close to zero.

(vi) It can be shown that by setting $\phi_{R C}=-\phi_{R} \phi_{C}$ we obtain an equivalence with the two-dimensional first-difference model of Kempton et al. (1994). We note, however, that an implication of this constraint is that for the Kempton et al. model, the pairwise variances between plots not in the same row or column of a replicate actually decrease with distance. Hence, we do not recommend the Kempton et al. model.

(vii) The LV model is a limiting case of the AR model when the correlation parameter, $\rho$ say, tends to one. Analyses of field trials using the AR model yield estimates of $\rho$ that are often close to one. For example, Patterson, in the discussion of Wilkinson et al. (1983), reported an average value of $\rho=0.94$ for the one-dimensional analysis of 244 U.K. cereal trials. More recently Costa e Silva, Dutkowski, and Gilmour (2001) carried out a two-dimensional analysis of a series of 12 forestry trials and obtained average values for the row and column correlation parameters of 0.87 and 0.79 , respectively, with ranges $(0.43-0.99)$ and $(0.17-0.98)$; however, these estimates could be lower if rows and columns had been included in their model.

(viii) Where the LV model is appropriate, no correlation parameters need to be estimated. This can help with model convergence, particularly in multistratum designs such as multiple-tree plot latinized row-column designs as used in forestry field trials.

Example 1. Williams, Matheson, and Harwood (2002, example 8.1) discussed the analysis of a latinized row-column design for $r=4$ replicates, each with $k=6$ rows and $s=$ 10 columns. Thus the overall dimension of the trial was $24 \times$ 10 plots. Each plot consisted of a $5 \times 5$ square of trees. The treatments were 59 provenances of Casuarina equisetifolia (the other proposed provenance was not planted out). Further details of this trial are given by Williams et al. (2002) who include a table of plot means for the variate $d b h$ (diameter at breast height) and the latinized row-column analysis of $d b h$. In this analysis replicates, provenances, and long columns are specified as fixed effects, with rows and columns within replicates as random effects. The inclusion of the random terms reduced the deviance by 16.95 (2 degrees of freedom). 
Sequentially adding spatial terms to the latinized rowcolumn model showed that the term $\phi_{R C}$ was not needed. Furthermore, due to the plots being square, estimated values for $\phi_{R}$ and $\phi_{C}$ were similar and so were collapsed into a single parameter $\phi$, say. The change in deviance for this reduced $\mathrm{LV}+\mathrm{RC}$ model was 6.71 (1 degree of freedom). Hence, for this example the $\mathrm{LV}+\mathrm{RC}$ analysis showed a significant improvement over the $\mathrm{RC}$ analysis. There was, however, very close agreement between the estimated provenance means from the $\mathrm{RC}$ and $\mathrm{LV}+\mathrm{RC}$ analyses.

\section{Construction of Resolvable Spatial Row-Column Designs}

Martin et al. (1993) investigated the construction of robust spatial block designs using the LV framework and developed some general construction principles. They considered designs that are efficient for the LV + IB model with spatial parameter $\phi$ in the range 0 to $5\left(k^{2}-1\right) / 6$ (note that the $\psi$ parameter of Martin et al. [1993] equals $\left.\phi\left(k^{2}-1\right) / 6\right)$. This range accords with the observations of Williams (1986) on the U.K. cereal trials where the average value of $\phi$ was 2.5 with average block size $k=6.3$. Williams (1985) derived a spatial optimality criterion appropriate for $\phi$ values in this range, which can be used to construct spatial designs consistent with the Martin et al. principles. The criterion is based on a fixed block effects (or intrablock) model so block effects can be eliminated by pre- and postmultiplying the $\mathrm{LV}+\mathrm{IB}$ variance matrix by the sweep matrix for blocks (de Hoog, Speed, and Williams, 1990).

Having defined the form of the $\mathrm{LV}+\mathrm{RC}$ model we can now extend the approaches of Martin et al. (1993) and Williams (1985) to two dimensions. We develop a criterion for the construction of efficient resolvable spatial row-column designs by defining a form for the treatment information matrix of an intrarow and intracolumn LV model. For the purpose of developing a convenient neighbor average efficiency factor $E$, we observe that the final term in (2), which is the row-column spatial interaction term, also contains information on treatment relationships within both rows and columns. Hence, we only need to retain this term in the development of a form for $E$, so following Williams (1985) the intrarow and intracolumn treatment information matrix is

$$
A=r I-X^{\prime}\left(I_{r} \otimes W_{R} \otimes W_{C}\right) X,
$$

where $W_{C}=\left(I-K_{k}\right) U_{k}\left(I-K_{k}\right)+K_{k}$ and so has $(i, j)$ th element

$$
\left\{6 /\left[k\left(k^{2}-1\right)\right]\right\}\left(\sum_{h=0}^{i-1} h+\sum_{h=0}^{k-j} h\right) \quad(1 \leq i \leq j \leq k) .
$$

The expression $W_{R}$ has a similar form with $k$ replaced by $s$. The expressions $W_{R}$ and $W_{C}$ are called weight matrices for the LV + RC model. Note that replacing $W_{R}$ by $K_{s}$ gives equation (16) of Williams (1985) for the one-dimensional LV + IB design with columns as blocks.

The spatial average efficiency factor $E$ is defined in the standard way as the harmonic mean of the nonzero eigenvalues of $A / r$, that is,

$$
E=\frac{v-1}{r \operatorname{trace}\left(A^{+}\right)}
$$

where $A^{+}$is the Moore-Penrose inverse of $A$ (John and Williams, 1995, p. 31). An upper bound for $E$, given when all the nonzero eigenvalues of $A / r$ are equal, is

$$
U=\frac{v-t}{v-1}
$$

where $t=\operatorname{trace}\left(W_{R}\right) \cdot \operatorname{trace}\left(W_{C}\right)$.

The spatial average efficiency factor can be used as a criterion in a computer search for efficient resolvable spatial rowcolumn designs. The efficiency factor update methods used by John and Whitaker (2000) for block and row-column designs can be extended to cater for one- and two-dimensional spatial designs. This has been implemented in the design generation package CycDesigN (Whitaker et al., 2002). In searching for efficient spatial designs we also need to ensure that the average efficiency factor of the resolvable row-column design, along with the average efficiency factors of the component row design and column design, are optimal or nearoptimal. Then, at the time of analysis, if the spatial structure or any of the blocking structures are not contributing to the model, we can safely revert to a simpler structure in (2). This also ensures that principle (a) of Martin et al. (1993) for robust designs is addressed, namely that the constructed designs are binary for both rows and columns. The remainder of principle (a), that is, the end design (plots 1 and $k$ ) and the interior design (plots 2 to $k-1$ ) should approximately contain each treatment equally often, is more difficult to satisfy in rowcolumn designs than in block designs, especially for smaller numbers of treatments. This essentially stems from the fact that there is a much higher proportion of edge plots in a two-dimensional arrangement of plots. Optimizing the spatial average efficiency factor does address this requirement in conjunction with the neighbor properties of treatments within rows and columns (principles (b) and (c)).

Example 2. Consider an $\mathrm{LV}+\mathrm{RC}$ design for $v=42$ treatments and $r=3$ replicates each with $k=6$ rows and $s=7$ columns. Using the software package CycDesigN, we obtain the design in Table 1 with spatial average efficiency factor $E=$ 0.9204 (upper bound $U=0.9268$ ). Some of the spatial features of the design can be seen by noting the immediate neighbors of treatments. For instance, treatment 1 has as row neighbors treatments $8,13,26$, and 41 , while the column neighbors are treatments $2,16,28,32$, and 38 ; a total of nine neighbors. Two treatments have only eight neighbors, namely treatments 19 and 38. These treatments occur in the corner position of one replicate and on the edges of the other two replicates. In fact, there are a total of nine treatments that fall in corner/edge positions of all three replicates. While having a number of treatments in these positions appears to violate principle (a) of Martin et al. (1993) the situation is slightly more complex with row-column designs. For instance, in replicate 3 of the design in Table 1 if columns 1 and 3, columns 5 and 7 , rows 1 and 3 , and rows 5 and 6 were interchanged then a design will be obtained with no treatments occurring in more than two replicates in the corner/edge positions. However, treatments 25 and 36 both occur in corner positions in two replicates and hence have eight neighbors. The neighbor features of both designs are very similar, as are the average efficiency factors ( $E=0.9202$ for the second design). 
Table 1

Row-column $L V$ design for 42 treatments

\begin{tabular}{|c|c|c|c|c|c|c|c|c|c|c|c|c|c|c|c|c|c|c|c|c|c|}
\hline \multirow[b]{2}{*}{ Column } & \multicolumn{7}{|c|}{ Replicate 1} & \multicolumn{7}{|c|}{ Replicate 2} & \multicolumn{7}{|c|}{ Replicate 3} \\
\hline & 1 & 2 & 3 & 4 & 5 & 6 & 7 & 1 & 2 & 3 & 4 & 5 & 6 & 7 & 1 & 2 & 3 & 4 & 5 & 6 & 7 \\
\hline Row 1 & 19 & 34 & 27 & 3 & 15 & 12 & 2 & 38 & 34 & 26 & 33 & 7 & 10 & 11 & 20 & 26 & 23 & 37 & 32 & 27 & 35 \\
\hline 2 & 40 & 4 & 7 & 20 & 21 & 33 & 18 & 1 & 13 & 15 & 35 & 5 & 3 & 18 & 10 & 22 & 16 & 18 & 42 & 8 & 12 \\
\hline 3 & 23 & 35 & 17 & 29 & 22 & 28 & 38 & 32 & 20 & 17 & 41 & 8 & 24 & 19 & 28 & 4 & 15 & 39 & 7 & 13 & 24 \\
\hline 4 & 10 & 14 & 31 & 39 & 32 & 5 & 42 & 12 & 30 & 6 & 39 & 23 & 21 & 16 & 1 & 41 & 14 & 34 & 30 & 29 & 40 \\
\hline 5 & 13 & 6 & 16 & 36 & 11 & 37 & 41 & 4 & 31 & 42 & 25 & 2 & 37 & 29 & 2 & 5 & 36 & 17 & 25 & 21 & 11 \\
\hline 6 & 25 & 8 & 1 & 26 & 9 & 30 & 24 & 36 & 22 & 40 & 27 & 9 & 14 & 28 & 6 & 19 & 33 & 9 & 3 & 38 & 31 \\
\hline
\end{tabular}

The construction procedure also ensures that the resolvable row-column design is efficient. The average efficiency factor is 0.6941 , compared with an upper bound of 0.7059 , for both of the designs considered above.

\section{Comparison of Spatial Designs}

Spatial designs that are efficient for the two-dimensional AR model have been constructed for use in cereal variety trials (Coombes, Payne, and Lisboa, 1998; Eccleston and Chan, 1998). The AR model is assumed to be of the form (1) with variance matrix given by

$$
\begin{gathered}
V^{*}=\left(I_{n}-P_{G}\right)\left(I_{n}+\eta_{R} P_{R}+\eta_{C} P_{C}+\psi I_{r} \otimes D\left(\rho_{C}\right)\right. \\
\left.\otimes D\left(\rho_{R}\right)\right)\left(I_{n}-P_{G}\right),
\end{gathered}
$$

where $D\left(\rho_{C}\right)$ and $D\left(\rho_{R}\right)$ are $s \times s$ and $k \times k$ matrices with $(i, j)$ th elements given by $\rho_{C}^{|i-j|}$ and $\rho_{R}^{|i-j|}$, respectively. Fixed parameter values are then assigned to produce $V^{*}$ and designs constructed to minimize the average pairwise variance between treatments which can be written as $z(X)=$ trace $\left(X^{\prime} V^{*+} X\right)^{+} /(v-1)$. As noted earlier the LV structure is a limiting form of the AR structure as $\rho$ tends to one.

We can carry out a comparison of $\mathrm{LV}$ and AR constructed designs. For example, the design in Table 2 has been constructed (by one of the referees) to be efficient for the parameter values $\eta_{R}=35, \eta_{C}=60, \psi=10$, and $\rho_{R}=\rho_{C}=$ 0.5. We refer to the designs in Tables 1 and 2 as Designs 1 and 2, respectively. So if $X_{1}$ and $X_{2}$ are the treatment design matrices for Designs 1 and 2, we can use $R E=z\left(X_{2}\right) / z\left(X_{1}\right)$ to compare the relative efficiencies of designs for a range of parameter values in (4); this is done in Table 3. Parameter set 1 gives the variance structure used for the Table 2 design and the relative efficiency is less than one, meaning that Design 2 is more efficient than Design 1. Parameter set 2 reduces the emphasis on row and column effects; in other words it gives greater weight to the spatial component. Parameter set 3 is more typical for cereal trials with long thin plots, having a low correlation parameter in one direction. The remaining parameter sets have equal row and column correlation parameters and would be appropriate for square plots as, for example, in the case of many forestry trials. Parameter sets 4 and 5 have high correlation parameters and spatial variance component comparable in size to the independent error term or nugget effect, that is, the ratio of spatial variance to independent error is 1.0. The correlation parameters and spatial variance component are representative of the average results from the Costa e Silva et al. (2001) trials; there no row or column effects were fitted and so, for comparison, we have varied the size of the row and column components.

It is difficult to cover a wide range of parameter sets in Table 3 and as such to make general recommendations. It seems, however, that the LV model is efficient where the correlation is high in both dimensions and the spatial variance component is comparable with the independent error term. It is here that the AR structure approaches its limiting form.

\section{Discussion}

The formulation of the $\mathrm{LV}+\mathrm{RC}$ model provides a framework to develop a construction criterion for the evaluation of resolvable spatial row-column designs. This is facilitated by the fact that the variance matrix for the model can be written as a linear combination of known matrices. Furthermore, the design criterion does not require any prior values for variance and correlation parameters. The spatial average efficiency factor

Table 2

\begin{tabular}{|c|c|c|c|c|c|c|c|c|c|c|c|c|c|c|c|c|c|c|c|c|c|}
\hline \multirow[b]{2}{*}{ Column } & \multicolumn{7}{|c|}{ Replicate 1} & \multicolumn{7}{|c|}{ Replicate 2} & \multicolumn{7}{|c|}{ Replicate 3} \\
\hline & 1 & 2 & 3 & 4 & 5 & 6 & 7 & 1 & 2 & 3 & 4 & 5 & 6 & 7 & 1 & 2 & 3 & 4 & 5 & 6 & 7 \\
\hline Row 1 & 11 & 8 & 33 & 23 & 10 & 6 & 29 & 6 & 2 & 31 & 20 & 13 & 14 & 5 & 5 & 28 & 20 & 30 & 8 & 7 & 26 \\
\hline 2 & 39 & 28 & 17 & 4 & 2 & 21 & 38 & 42 & 36 & 26 & 19 & 35 & 25 & 38 & 2 & 19 & 14 & 12 & 39 & 31 & 37 \\
\hline 3 & 16 & 32 & 41 & 37 & 42 & 9 & 13 & 24 & 11 & 10 & 9 & 40 & 1 & 4 & 34 & 11 & 35 & 27 & 6 & 15 & 40 \\
\hline 4 & 1 & 7 & 19 & 5 & 3 & 36 & 18 & 30 & 22 & 33 & 18 & 16 & 28 & 27 & 18 & 4 & 41 & 3 & 25 & 10 & 22 \\
\hline 5 & 27 & 14 & 34 & 30 & 40 & 31 & 25 & 21 & 12 & 32 & 15 & 17 & 37 & 8 & 24 & 33 & 21 & 13 & 36 & 16 & 29 \\
\hline 6 & 26 & 24 & 12 & 35 & 20 & 22 & 15 & 3 & 23 & 34 & 39 & 7 & 29 & 41 & 17 & 42 & 23 & 1 & 32 & 38 & 9 \\
\hline
\end{tabular}

Row-column AR design for 42 treatments 
Table 3

Relative efficiencies ( $R E$ ) of the $L V$ design (Table 1) to the $A R$ design (Table 2) for different assumed variance structures

\begin{tabular}{lrrrrrr}
\hline \hline Parameter set & $\eta_{R}$ & $\eta_{C}$ & $\psi$ & $\rho_{R}$ & $\rho_{C}$ & $\mathrm{RE}$ \\
\hline 1 & 35 & 60 & 10 & 0.5 & 0.5 & 0.9567 \\
2 & 5 & 10 & 10 & 0.6 & 0.5 & 0.9645 \\
3 & 5 & 10 & 10 & 0.2 & 0.8 & 0.9609 \\
4 & 70 & 60 & 1 & 0.9 & 0.9 & 1.0434 \\
5 & 10 & 10 & 1 & 0.9 & 0.9 & 1.0337 \\
6 & 35 & 30 & 0.5 & 0.8 & 0.8 & 1.0379 \\
7 & 2 & 2 & 0.5 & 0.8 & 0.8 & 1.0154 \\
8 & 0 & 0 & 0.5 & 0.8 & 0.8 & 1.0054 \\
\hline
\end{tabular}

addresses the two-dimensional extension of the principles outlined by Martin et al. (1993) although we have found that these principles are more easily adhered to for larger treatment numbers.

The recent developments for updating the average efficiency factor in the construction of block and row-column designs (John and Whitaker, 2000) have been extended to accommodate one- and two-dimensional spatial structures. This amounts to incorporating the $\mathrm{LV}+\mathrm{RC}$ weight matrices $W_{R}$ and $W_{C}$ into the design generation algorithm. Other forms of weight matrices can also be used, such as exponential decay or integer weights, as described by Wild and Williams (1987). The former would be appropriate for an AR model as used by Eccleston and Chan (1998). An exponential decay matrix does, however, require the rate of decay to be specified in advance. Nevertheless, we have observed that efficient spatial designs are quite robust to the choice of decay parameter and indeed to the type of spatial weight matrix used. These options have been incorporated into the design generation package CycDesigN.

When replicates are contiguous, latinized designs are recommended (see John and Williams, 1995, p. 72). The use of $t$-latinized designs (John and Williams, 1998) is effective to ensure that the replicates of a treatment are spread across the long columns of the contiguous replicates. In our spatial construction approach, we would maintain the latinized structure when carrying out spatial improvement. It is interesting to note that if we use $L_{r k}$ to assign an LV spatial structure down the long column of $r k$ plots in a contiguous-replicate design then

$$
\left(I_{n}-P_{C}\right) L_{r k}\left(I_{n}-P_{C}\right)=\left(I_{n}-P_{C}\right) L_{k}\left(I_{n}-P_{C}\right) .
$$

In other words, in the construction of contiguous-replicate designs, restricting consideration to designs where the spatial structure only applies within replicates does not reduce the efficiency of those designs.

The LV + RC model facilitates an evaluation of the spatial analysis of resolvable field trials against conventional rowcolumn mixed-model analysis. The random parameters in the $\mathrm{LV}+\mathrm{RC}$ model all appear as coefficients to specific matrices, such as the projection matrices $P_{R}$ and $P_{C}$ for the blocking factors and the normalized spatial matrices $F_{R}, F_{C}$, and $F_{R C}$. Such a structure is amenable to a sequential fitting process based on the reduction in deviance for the fitted model. Thus, if the spatial terms are not needed in the model, or if the model is wrong, we can successively revert to a simpler model. Hence, the LV $+\mathrm{RC}$ model can never be worse than the RC model. The REML commands in GenStat provide a straightforward approach to the estimation process although, as the package checks for positive definiteness on each of the spatial matrices in the variance structure, it is necessary to carry out a reparameterization by adding $s P_{R}$ to $F_{R}$, and so on. This does not change the model; it just means that the coefficient $\eta_{R}$ for the term in $P_{R}$ is replaced by $\eta_{R}-\phi_{R}$, etc. Overall the estimated parameters are such that the variance matrix of the $\mathrm{LV}+\mathrm{RC}$ model is positive semidefinite.

Designs constructed for the LV + RC model are particularly suitable for square plots with high correlations between both rows and columns, and where the independent error term or nugget effect is comparable in size with the spatial variance component. Such is the case for many forestry trials associated with outbred populations. Hence, the $\mathrm{LV}+\mathrm{RC}$ model addresses at least one important class of field trials.

\section{ACKNOWLEDGEMENTS}

This work was partially supported by an award from the Marsden Fund of the Royal Society of New Zealand. We would like to thank the referees and an associate editor for valuable comments.

\section{REFERENCES}

Baird, D. and Mead, R. (1991). The empirical efficiency and validity of two neighbour models. Biometrics 47, 14731487.

Besag, J. and Kempton, R. (1986). Statistical analysis of field experiments using neighbouring plots. Biometrics 42, 231-251.

Cochran, W. G. and Cox, G. M. (1957). Experimental Design, 2nd edition. New York: Wiley.

Coombes, N., Payne, R., and Lisboa, P. (1998). Comparison of nested simulated annealing and reactive tabu search for efficient experimental designs with correlated data. COMPSTAT Proceedings in Computational Statistics, 5364.

Costa e Silva, J., Dutkowski, G. W., and Gilmour, A. R. (2001). Analysis of early tree height in forest genetic trials is enhanced by including a spatially correlated residual. Canadian Journal of Forest Research 31, 1887-1893.

Cullis, B. R. and Gleeson, A. C. (1991). Spatial analysis of field experiments - An extension to two dimensions. Biometrics 47, 1449-1460.

de Hoog, F. R., Speed, T. P., and Williams, E. R. (1990). On a matrix identity associated with generalized least squares. Linear Algebra and Applications 127, 449-456.

Eccleston, J. A. and Chan, B. S. P. (1998). Design algorithms for correlated data. COMPSTAT Proceedings in Computational Statistics, 41-52.

Gleeson, A. C. and Cullis, B. R. (1987). Residual maximum likelihood (REML) analysis of a neighbour model for field experiments. Biometrics 43, 277-288.

John, J. A. and Whitaker, D. (2000). Recursive formulae for the average efficiency factor in block and row-column 
designs. Journal of the Royal Statistical Society, Series B 62, 575-583.

John, J. A. and Williams, E. R. (1995). Cyclic and Computer Generated Designs. London: Chapman and Hall.

John, J. A. and Williams, E. R. (1998). $t$-Latinized designs. Australian and New Zealand Journal of Statistics 40, 111118.

Kempton, R. A., Seraphin, J. C., and Sword, A. M. (1994). Statistical analysis of two dimensional variation in variety yield trials. Journal of Agricultural Science 122, 335342 .

Martin, R. J. (1979). A subclass of lattice processes applied to a problem in planar sampling. Biometrika 66, 209-217.

Martin, R. J. and Eccleston, J. E. (1991). Efficient block designs for correlated observations. Australian Journal of Statistics 33, 299-311.

Martin, R. J., Eccleston, J. E., and Gleeson, A. C. (1993). Robust linear block designs for a suspected LV model. Journal of Statistical Planning and Inference 34, 433-450.

Papadakis, J. S. (1937). Méthode statistique pour des expériences sur champ. Bulletin de l'Institut d'Amélioration des Plantes à Salonique, No. 23.

Patterson, H. D. and Hunter, E. A. (1983). The efficiency of incomplete block designs in National List and Recommended List cereal variety trials. Journal of Agricultural Science 101, 427-433.
Welham, S. J. and Thompson, R. (1997). Likelihood ratio tests for fixed model terms using residual maximum likelihood. Journal of the Royal Statistical Society, Series B 59, 701-714.

Whitaker, D., Williams, E. R., and John, J. A. (2002). CycDesigN: A Package for the Computer Generation of Experimental Designs. Canberra: CSIRO Forestry and Forest Products.

Wild, P. R. and Williams, E. R. (1987). The construction of neighbour designs. Biometrika 74, 871-876.

Wilkinson, G. N., Eckert, S. R., Hancock, T. W., and Mayo, O. (1983). Nearest neighbour analysis of field experiments (with discussion). Journal of the Royal Statistical Society, Series B 45, 151-211.

Williams, E. R. (1985). A criterion for the construction of optimal neighbour designs. Journal of the Royal Statistical Society, Series B 47, 489-497.

Williams, E. R. (1986). A neighbour model for field experiments. Biometrika 73, 279-287.

Williams, E. R., Matheson, A. C., and Harwood, C. E. (2002). Experimental Design and Analysis for Tree Improvement, 2nd edition. Melbourne: CSIRO.

Received June 2003. Revised March 2005. Accepted March 2005. 